DESY 06-103

SI-HEP-2006-07

hep-ph/0607127

July 2006

\title{
NLO Event Generation for Chargino Production at the ILC
}

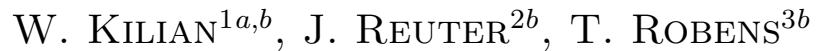 \\ ${ }^{a}$ Fachbereich Physik, University of Siegen, D-57068 Siegen, Germany \\ ${ }^{b}$ Deutsches Elektronen-Synchrotron DESY, D-22603 Hamburg, Germany
}

\begin{abstract}
We present a Monte-Carlo event generator for simulating chargino pairproduction at the International Linear Collider (ILC) at next-to-leading order in the electroweak couplings. By properly resumming photons in the soft and collinear regions, we avoid negative event weights, so the program can simulate physical (unweighted) event samples. Photons are explicitly generated throughout the range where they can be experimentally resolved. Inspecting the dependence on the cutoffs separating the soft and collinear regions, we evaluate the systematic errors due to soft and collinear approximations. In the resummation approach, the residual uncertainty can be brought down to the per-mil level, coinciding with the expected statistical uncertainty at the ILC.
\end{abstract}

\footnotetext{
${ }^{1}$ wolfgang.kilian@desy.de

2 juergen.reuter@desy.de

${ }^{3}$ tania.robens@desy.de
} 


\section{Introduction}

The MSSM, the minimal supersymmetric (SUSY) extension of the Standard Model (SM), is a promising candidate for a theory of electroweak interactions [1. In this model, the Higgs sector is stabilized against power divergences in radiative corrections, proton stability suggests a discrete symmetry that provides us with a dark-matter particle, and the renormalizationgroup evolution of couplings is precisely consistent with gauge-coupling unification (GUT) at an energy scale of the order $10^{16} \mathrm{GeV}$.

A solid prediction of the MSSM is the existence of charginos $\tilde{\chi}_{1}^{ \pm}, \tilde{\chi}_{2}^{ \pm}$, the superpartners of the $W^{ \pm}$and the charged-Higgs $H^{ \pm}$bosons. In GUT models their masses tend to be near the lower edge of the superpartner spectrum, since the absence of strong interactions precludes large positive renormalizations of their effective masses. Thus, if any superpartners are accessible in $e^{+} e^{-}$collisions at a first-phase ILC with c.m. energy of $500 \mathrm{GeV}$, the lighter chargino $\tilde{\chi}_{1}^{ \pm}$ is likely to be pair-produced with a sizable cross section. In many models, including popular supergravity-inspired scenarios such as SPS1a/SPS1a' 2, the second chargino $\tilde{\chi}_{2}^{ \pm}$will also be accessible at the ILC, at least if the c.m. energy is increased to about $1 \mathrm{TeV}$. Similar arguments hold for the neutralinos, the superpartners of neutral gauge and Higgs bosons. The lightest neutralino is possibly the lightest superpartner (LSP) and therefore the dark-matter particle present in the MSSM.

The precise measurement of the chargino parameters (masses, mixing of $\tilde{\chi}_{1}^{ \pm}$with $\tilde{\chi}_{2}^{ \pm}$, and couplings) is a key for uncovering any of the fundamental properties of the MSSM that we have mentioned above. These values give a handle for proving supersymmetry in the Higgs and gauge-boson sector and thus the cancellation of power divergences. Charginos decay either directly or via short cascades into the LSP, and a precise knowledge of masses and mixing parameters in the chargino/neutralino sector is the most important ingredient for predicting the dark-matter content of the universe. Finally, the high-scale evolution of their mass parameters should point to a particular supersymmetry-breaking scenario, if the context of a GUT model is assumed (cf. [3]). In all these cases, a knowledge of parameters with at least percent-level accuracy is necessary.

At the LHC, this is a difficult task since charginos are accessible mainly in complicated decay cascades of colored superpartners with substantial background, while direct pair-production is suppressed 4]. The ILC provides much cleaner production channels and decay signatures with low background, so the required precision will be available at the ILC [56]. To match this experimental accuracy, there is obvious need for theoretical predictions with next-to-leading order (NLO) accuracy in the electroweak couplings. The predictions have to be implemented in the simulation tools that are actually used in the experimental analyses (e.g. see [7]).

At leading order (LO), chargino production at the ILC is given by the tree-level diagrams in Fig. 11, and events can be generated using the narrow-width approximation where all processes are factorized in on-shell $2 \rightarrow 2$ production and a cascade of on-shell $1 \rightarrow n$ decay processes. The helicity amplitudes can be expressed in analytic form (cf. [8]), and the process is available in various computer codes [9].

The NLO corrections include ${ }^{1}$ (i) loop corrections to the SUSY production and decay pro-

\footnotetext{
${ }^{1}$ This describes the multiple-pole approximation [10]; recent complete NLO calculations in the SM [11] have
} 


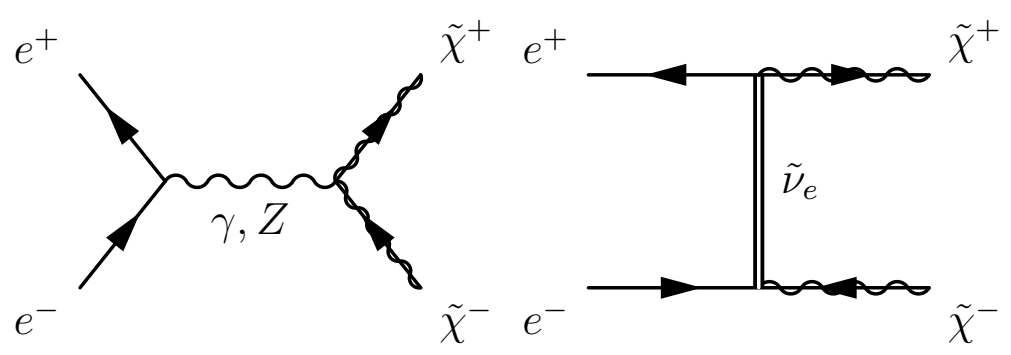

Figure 1: Feynman graphs for chargino pair production at the ILC.

\begin{tabular}{c|cc}
\hline & Mass & Width \\
\hline$\tilde{\chi}_{1}^{+}$ & $183.7 \mathrm{GeV}$ & $0.077 \mathrm{GeV}$ \\
$\tilde{\chi}_{2}^{+}$ & $415.4 \mathrm{GeV}$ & $3.1 \mathrm{GeV}$ \\
\hline
\end{tabular}

Table 1: Chargino masses and widths for the SUSY parameter set SPS1a'.

cesses, (ii) nonfactorizable, but maximally resonant photon exchange between production and decay, (iii) real radiation of photons, (iv) off-shell kinematics for the signal process, (v) irreducible background from all other multi-particle SUSY processes, and (vi) reducible, but experimentally indistinguishable background from Standard-Model (SM) processes. So far, no calculation and simulation code provides all NLO pieces for a process involving SUSY particles.

In Ref. [12, three computer codes have been presented and verified against each other that simulate off-shell multi-particle processes at tree-level, both for the SM and the MSSM. As generators of unweighted SUSY event samples, they thus cover (iv), (v), and (vi). In particular, the program described in this paper is implemented as an extension to the WHIZARD event generator [13. With beamstrahlung, resummed initial-state radiation, arbitrary polarization modes and standard parton-shower and hadronization interfaces being included, this generator is well suited for ILC physics studies.

In this paper, we describe the extension of the tree-level simulation of chargino production at the ILC by radiative corrections to the on-shell process, i.e., we consider (i) in the above list and consistently include real photon radiation (iii). This is actually a useful approximation since in many MSSM scenarios charginos, in particular $\tilde{\chi}_{1}^{ \pm}$, are quite narrow (cf. Table 1), so nonfactorizable contributions are significantly suppressed and decay corrections can be separated from the corrections to the production process.

We emphasize that for the simulation of physical (i.e., unweighted) event samples, it is essential that the effective matrix elements are positive semidefinite over the whole accessible phase space. The QED part of radiative corrections does not meet this requirement in some phase space regions. Methods for dealing with this problem have been developed in the LEP1 era [14. While these methods are also applicable for the ILC situation, they need a thorough reconsideration since the ILC precision actually exceeds the one achieved in LEP experiments.

explicitly verified the validity of this approximation in the signal region. 


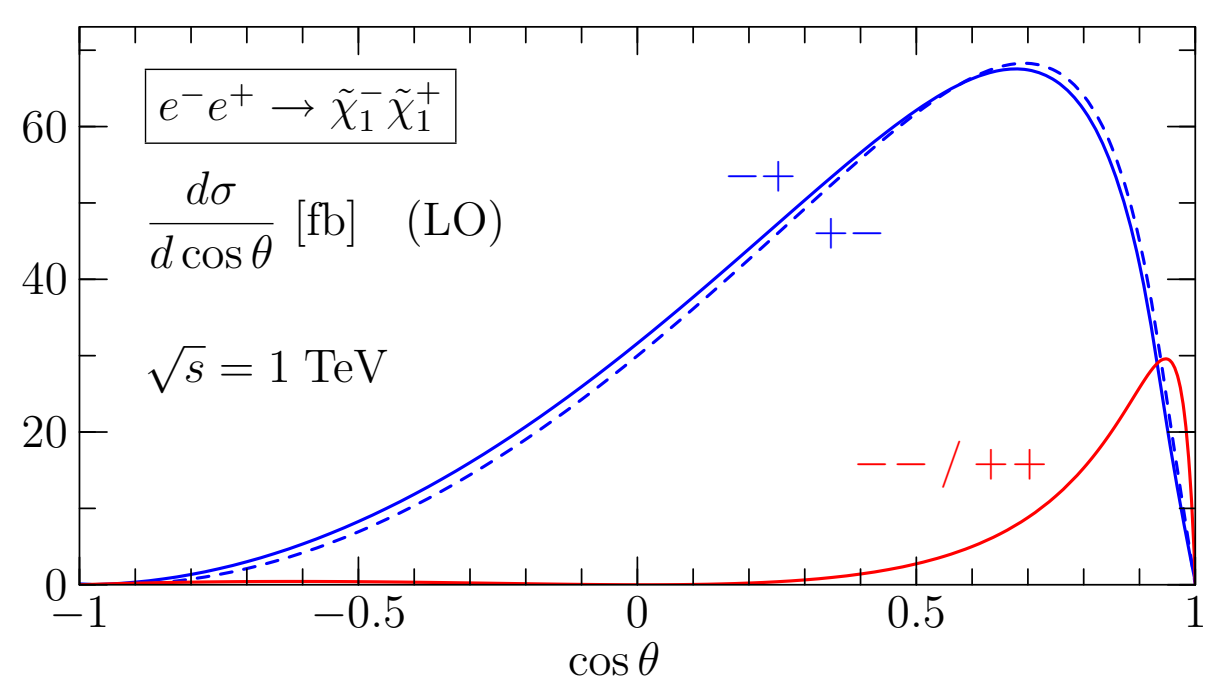

Figure 2: Chargino pair production at the ILC: Dependence of the differential distribution in polar angle $\cos \theta$ between $e^{-}$and $\tilde{\chi}_{1}^{-}$for different helicity combinations. The labels indicate $\tilde{\chi}_{1}^{-}$ and $\tilde{\chi}_{1}^{+}$helicity; the electron/positron helicity is fixed to -+ .

\section{Fixed-Order Simulation of Chargino Production}

\subsection{Lowest Order}

In the MSSM, the charginos $\tilde{\chi}_{1}^{ \pm}, \tilde{\chi}_{2}^{ \pm}$are mixtures of weak gauginos $\tilde{w}^{ \pm}$and higgsinos $\tilde{h}^{ \pm}$. The production processes in $e^{+} e^{-}$collisions are thus connected by SUSY transformations to $e^{+} e^{-} \rightarrow W^{+} W^{-}$and $e^{+} e^{-} \rightarrow H^{+} H^{-}$; the contributing Feynman diagrams consist of $s$-channel $e^{+} e^{-}$annihilation via $Z$ and photon and $t$-channel exchange of an electron-sneutrino (Fig. 1). Since no massless particles are exchanged in the $t$-channel, the electron mass can be neglected at tree level throughout the phase space. The square of the absolute value of the matrix element, integrated over the phase space $\Gamma$ which is parameterized by production angles $\theta, \phi$, defines the Born cross section $\sigma_{\text {Born }}$ :

$$
\sigma_{\text {Born }}(s)=\int d \Gamma_{2}\left|\mathcal{M}_{\text {Born }}(s, \cos \theta)\right|^{2}
$$

We suppress the dependence on particle masses $M_{\tilde{\chi}}, M_{Z}$, etc.

At the ILC, the possibility of polarizing electrons and positrons in the initial state will allow for separately measuring individual (squared) helicity amplitudes. Selecting a standard MSSM parameter point SPS1a' (cf. App. C] and Table 1) and a collider energy of 1 TeV, in Fig. 2 we display the angular dependence of the cross section for the dominant helicity combinations with $\tilde{\nu}$ exchange in the $t$ channel. As the amplitudes are $\propto(1 \pm \cos \theta), \sin \theta$ respectively, they can become zero for $\theta= \pm \pi, 0$. 


\subsection{NLO Corrections}

The one-loop corrections to the process $e^{-} e^{+} \rightarrow \tilde{\chi}_{i}^{-} \tilde{\chi}_{j}^{+}$with $j=1,2$ have been computed in Ref. [15, using the FeynArts/FormCalc package [16] for the evaluation of one-loop Feynman diagrams in the MSSM. An independent calculation with consistent numerical results has been presented in [17]. These calculations include the complete set of virtual diagrams contributing to the process with both SM and SUSY particles in the loop. The collinear singularity for photon radiation off the incoming electron and positron is regulated by the finite electron mass $m_{e}$. As an infrared regulator, the calculation introduces a fictitious photon mass $\lambda$. The interference of these diagrams with the Born term defines the 'virtual' contribution

$$
\sigma_{\text {virt }}\left(s, \lambda^{2}, m_{e}^{2}\right)=\int d \Gamma_{2}\left[2 \operatorname{Re}\left(\mathcal{M}_{\text {Born }}(s)^{*} \mathcal{M}_{1-\text { loop }}\left(s, \lambda^{2}, m_{e}^{2}\right)\right)\right] .
$$

The dependence on the fictitious parameter $\lambda$ is eliminated by neglecting contributions proportional to powers of $\lambda$ and adding real photon radiation with energy $E_{\gamma}<\Delta E_{\gamma}$, where $E_{\gamma}$ is defined in some reference frame, usually the c.m. frame. Hence, the residual logarithmic dependence on $\lambda$ is cancelled in favor of a logarithmic dependence on $\Delta E_{\gamma}$. This correction can be expressed as a universal factor $f_{\text {soft }}\left(\frac{\Delta E_{\gamma}}{\lambda}\right)$ (19). The 'virtual + soft' contribution $\sigma_{\mathrm{v}+\mathrm{s}}\left(s, \Delta E_{\gamma}, m_{e}^{2}\right)$ is thus given by

$$
\sigma_{\mathrm{v}+\mathrm{s}}\left(s, \Delta E_{\gamma}, m_{e}^{2}\right)=\int d \Gamma_{2}\left[f_{\text {soft }}\left(\frac{\Delta E_{\gamma}}{\lambda}\right)\left|\mathcal{M}_{\text {Born }}(s)\right|^{2}+2 \operatorname{Re}\left(\mathcal{M}_{\text {Born }}(s)^{*} \mathcal{M}_{1 \text {-loop }}\left(s, \lambda^{2}, m_{e}^{2}\right)\right)\right] .
$$

In a real experiment, there is always a finite energy resolution $\Delta E_{\gamma}^{\exp }$ for photons, and combining soft and virtual photons below this cutoff is justified. For the simulation one would choose $\Delta E_{\gamma} \leq \Delta E_{\gamma}^{\exp }$.

This result is complemented by the 'hard' contribution $\sigma_{2 \rightarrow 3}\left(s, \Delta E_{\gamma}, m_{e}^{2}\right)$, i.e., the realradiation process $e^{-} e^{+} \rightarrow \tilde{\chi}_{i}^{-} \tilde{\chi}_{j}^{+} \gamma$ integrated over photon phase space down to the energy resolution $\Delta E_{\gamma}$ :

$$
\sigma_{2 \rightarrow 3}\left(s, \Delta E_{\gamma}, m_{e}^{2}\right)=\int_{\Delta E_{\gamma}} d \Gamma_{3}\left|\mathcal{M}_{2 \rightarrow 3}\left(s, m_{e}^{2}\right)\right|^{2} .
$$

The sum, which can be expressed as a total cross section or, e.g., as a differential distribution in the chargino polar angle $\theta$, should not depend on the photon-energy cutoff:

$$
\sigma_{\text {tot }}\left(s, m_{e}^{2}\right)=\sigma_{\text {Born }}(s)+\sigma_{\mathrm{v}+\mathrm{s}}\left(s, \Delta E_{\gamma}, m_{e}^{2}\right)+\sigma_{2 \rightarrow 3}\left(s, \Delta E_{\gamma}, m_{e}^{2}\right)
$$

However, the dependence on $\Delta E_{\gamma}$ cancels only approximately since positive powers of $\Delta E_{\gamma}$ are neglected in the $\mathrm{v}+\mathrm{s}$ term but not in the $2 \rightarrow 3$ process.

\subsection{Collinear Photons}

While photons with large energy and large angle can be experimentally resolved and must be explicitly generated by the Monte-Carlo simulation program, photons collinear to the incoming electrons cannot be detected. (Since the outgoing charginos have substantial mass, a collinear 
approximation for final-state radiation is not needed.) As usual, we break down the (hard) $2 \rightarrow 3$ cross section into a collinear and a non-collinear part, separated at a photon acollinearity angle $\Delta \theta_{\gamma}$ relative to the incoming electron or positron:

$$
\sigma_{2 \rightarrow 3}\left(s, \Delta E_{\gamma}, m_{e}^{2}\right)=\sigma_{\text {hard,non-coll }}\left(s, \Delta E_{\gamma}, \Delta \theta_{\gamma}\right)+\sigma_{\text {hard,coll }}\left(s, \Delta E_{\gamma}, \Delta \theta_{\gamma}, m_{e}^{2}\right),
$$

where in the non-collinear part the electron mass can be neglected. The last term is approximated by convoluting the Born cross section with a structure function $f\left(x ; \Delta \theta_{\gamma}, \frac{m_{e}^{2}}{s}\right)$, with $x=1-2 E_{\gamma} / \sqrt{s}$ being the energy fraction of the electron after radiation,

$$
\begin{aligned}
\sigma_{\text {hard,coll }}\left(s, \Delta E_{\gamma}, \Delta \theta_{\gamma}, m_{e}^{2}\right) & =\int_{\Delta E_{\gamma}, \Delta \theta_{\gamma}} d \Gamma_{3}\left|\mathcal{M}_{2 \rightarrow 3}\left(s, m_{e}^{2}\right)\right|^{2} \\
& =\int_{0}^{x_{0}} d x f\left(x ; \Delta \theta_{\gamma}, \frac{m_{e}^{2}}{s}\right) \int d \Gamma_{2}\left|\mathcal{M}_{\text {Born }}\left(x s, m_{e}^{2}\right)\right|^{2} .
\end{aligned}
$$

The structure function $f\left(x ; \Delta \theta_{\gamma}, \frac{m_{e}^{2}}{s}\right)$ contains two pieces $f^{+}, f^{-}$(2123) that correspond to helicity conservation and helicity flip, respectively; each one is convoluted with the corresponding matrix element. The cutoff $\Delta E_{\gamma}$ is replaced by $x_{0}=1-2 \Delta E_{\gamma} / \sqrt{s}$. In this approximation, positive powers of $\Delta \theta_{\gamma}$ are neglected.

\subsection{Simulation}

Combining the above, the cross section is given by

$$
\begin{aligned}
\sigma_{\text {tot }}\left(s, m_{e}^{2}\right)= & \int d x f_{\text {eff }}\left(x_{1}, x_{2} ; \Delta E_{\gamma}, \Delta \theta_{\gamma}, \frac{m_{e}^{2}}{s}\right) \int d \Gamma_{2}\left|\mathcal{M}_{\mathrm{eff}}\left(s, x_{1}, x_{2} ; m_{e}^{2}\right)\right|^{2} \\
& +\int_{\Delta E_{\gamma}, \Delta \theta_{\gamma}} d \Gamma_{3}\left|\mathcal{M}_{2 \rightarrow 3}(s)\right|^{2}
\end{aligned}
$$

where we define

$$
\begin{aligned}
f_{\mathrm{eff}}\left(x_{1}, x_{2} ; \Delta E_{\gamma}, \Delta \theta_{\gamma}, \frac{m_{e}^{2}}{s}\right)= & \delta\left(1-x_{1}\right) \delta\left(1-x_{2}\right) \\
& +\delta\left(1-x_{1}\right) f\left(x_{2} ; \Delta \theta_{\gamma}, \frac{m_{e}^{2}}{s}\right) \theta\left(x_{0}-x_{2}\right) \\
& +f\left(x_{1} ; \Delta \theta_{\gamma}, \frac{m_{e}^{2}}{s}\right) \delta\left(1-x_{2}\right) \theta\left(x_{0}-x_{1}\right)
\end{aligned}
$$

and

$$
\begin{aligned}
\left|\mathcal{M}_{\text {eff }}\left(s, x_{1}, x_{2} ; m_{e}^{2}\right)\right|^{2}= & {\left.\left[1+f_{\text {soft }}\left(\Delta E_{\gamma}, \lambda^{2}\right) \theta\left(x_{1}, x_{2}\right)\right)\right]\left|\mathcal{M}_{\text {Born }}(s)\right|^{2} } \\
& +2 \operatorname{Re}\left[\mathcal{M}_{\text {Born }}(s) \mathcal{M}_{1-\text { loop }}\left(s, \lambda^{2}, m_{e}^{2}\right)\right] \theta\left(x_{1}, x_{2}\right)
\end{aligned}
$$

with $\theta\left(x_{1}, x_{2}\right) \equiv \theta\left(x_{1}-x_{0}\right) \theta\left(x_{2}-x_{0}\right)$.

This structure is suitable for implementing it into an event generator. In WHIZARD, for instance, there is an interface for arbitrary structure functions $f\left(x_{1}, x_{2}\right)$ that can be convoluted with the Born squared matrix element. We insert the above effective radiator function $f_{\text {eff }}$ as 
a 'user-defined' structure function and replace the Born matrix element as computed by the matrix-element generator, O’Mega [18, by the effective matrix element defined above. The latter is computed by a call to the FormCalc-generated routine.

In order to account for the $\delta$-function part contained in the radiator function, for the MonteCarlo sampling of $x$ values the $x_{i}$ range is separated into two regions each, one for $x_{i}<x_{0}$ and the other one for $x_{i}>x_{0}$. For each $x_{i}$, the first region is mapped such as to maximize the efficiency of event generation. If the sampled point ends up in the second region, $x_{i}$ is set equal to 1 before the matrix element is evaluated as demanded by the $\delta$ function. The relative weight of the two regions is given by

$$
w\left(x>x_{0}\right): w\left(x<x_{0}\right)=1: \int_{0}^{x_{0}} d x f\left(x ; \Delta \theta_{\gamma}, \frac{m_{e}^{2}}{s}\right) .
$$

For a consistent first-order calculation, we have to avoid the radiation of two (collinear) photons. Therefore, the radiator function $f_{\text {eff }}$ is zero in the region with $x_{1}<x_{0}$ and $x_{2}<x_{0}$, and in the $2 \rightarrow 3$ process, no convolution with structure functions is applied.

Implementing this algorithm in WHIZARD, we construct an unweighted event generator. With separate runs for the $2 \rightarrow 2$ and $2 \rightarrow 3$ parts, the program first adapts the phase space sampling and calculates a precise estimate of the cross section. The built-in routines apply event rejection based on the effective weight and thus generate unweighted event samples.

For the $2 \rightarrow 2$ part convoluted with a structure function, WHIZARD can optionally represent the missing collinear energy by a real photon in the event, with $p_{T}$ generated according to the correct logarithmic distribution up to the cutoff angle $\Delta \theta_{\gamma}$. Thus, if there is any energy available for radiation, the actual events contain a photon in addition to the chargino pair regardless whether the event has been generated in the $2 \rightarrow 2$ or $2 \rightarrow 3$ part.

On the technical side, for the actual implementation we have carefully checked that all physical parameters and, in particular, the definition of helicity states are correctly matched between the conventions [19] used by O'Mega and WHIZARD [20], and those used by FormCalc (cf. e.g. 21]).

\subsection{Where this Approach Fails}

Numerically, the modified WHIZARD code reproduces the total cross section at fixed next-toleading order in $\alpha$ as presented in Ref. [15. In principle, this makes the NLO result available for physics simulation. However, in the soft-photon region the fixed-order approach runs into the well-known problem of negative event weights 2223 .

While for any fixed helicity combination and chargino scattering angle the differential cross section is positive if we include the virtual contribution and integrate real soft photons up to a finite cutoff $\Delta E_{\gamma}$, the $2 \rightarrow 2$ part of the NLO-corrected squared matrix element is positive definite by itself only if $\Delta E_{\gamma}$ is sufficiently large, say $\frac{\Delta E_{\gamma}}{\sqrt{s}}=10^{-2}$ (i.e., $\Delta E_{\gamma}=10 \mathrm{GeV}$ for $\sqrt{s}=1 \mathrm{TeV})$. If we lower the cutoff, say $\frac{\Delta E_{\gamma}}{\sqrt{s}}<10^{-3}$, for each helicity combination the effective $2 \rightarrow 2$ matrix element becomes negative within some range of scattering angle, compensating the $2 \rightarrow 3$ squared matrix element that for such a small cutoff overshoots the total NLO differential cross section. This is illustrated in Fig. 3 . 

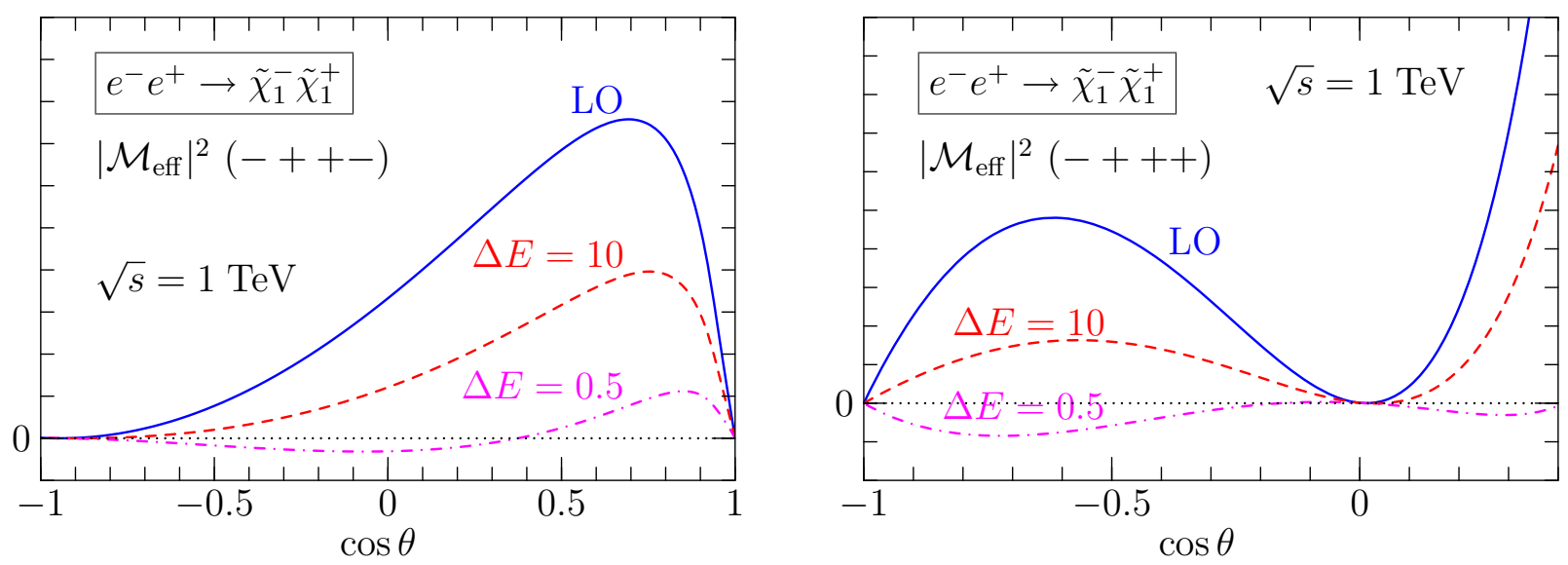

Figure 3: Effective squared matrix element (arbitrary units) for $e^{-} e^{+} \rightarrow \tilde{\chi}_{1}^{-} \tilde{\chi}_{1}^{+}$as a function of the polar scattering angle $\theta$ at $\sqrt{s}=1 \mathrm{TeV}$. Left figure: Helicity combination -++- ; right figure: -+++ . Solid line: Born term; dashed: including virtual and soft contributions for $\Delta E_{\gamma}=10 \mathrm{GeV}$; dash-dotted: same with $\Delta E_{\gamma}=0.5 \mathrm{GeV}$.

However, the experimental resolution can be better than that, and for large $\Delta E_{\gamma}$ power corrections to the soft approximation are not completely negligible; therefore we are interested in letting $\Delta E_{\gamma} \rightarrow 0$. To obtain unweighted event samples in the presence of negative-weight events, an ad-hoc approach is to simply drop such events before proceeding further. The numerical consequences of such a truncation are discussed below. ${ }^{2}$

Instead of slicing phase space and introducing a separate treatment of the soft and collinear regions, an alternative approach uses subtractions in the integrand to eliminate the singularities before integration 24]; the subtracted pieces are integrated analytically and added back or cancelled against each other where possible. This method does not require technical cutoffs and therefore allows to get rid of cutoff-induced artefacts in the result. Unfortunately, the subtracted integrands do not necessarily satisfy positivity conditions either, so the transformation into an unweighted Monte-Carlo generator is not straightforward. (Of course, the method may be used to construct a weighted event generator.) We do not consider this method in the present paper.

\section{Resumming Photons}

\subsection{Leading Logarithms}

The shortcomings of the fixed-order approach described above are associated with the softcollinear region $E_{\gamma}<\Delta E_{\gamma}, \theta_{\gamma}<\Delta \theta_{\gamma}$, where the appearance of double logarithms $\frac{\alpha}{\pi} \ln \frac{E_{\gamma}^{2}}{s} \ln \theta_{\gamma}$

\footnotetext{
${ }^{2}$ Strictly speaking, events with a weight that can become negative do not preclude detector simulation and further analysis, but the event samples containing them are not a possible outcome of a physical experiment. In particular, simulating the detector response to such events is a waste of resources. If a method is available that eliminates them, it is the preferable choice.
} 
invalidates the perturbative series. However, in that region higher-order radiation can be resummed [25]. The exponentiated structure function $f_{\text {ISR }}$ [26] given in Eq. (24) that resums initial-state radiation,

$$
\sigma_{\mathrm{Born}+\mathrm{ISR}}\left(s, \Delta \theta_{\gamma}, m_{e}^{2}\right)=\int d x f_{\mathrm{ISR}}\left(x ; \Delta \theta_{\gamma}, \frac{m_{e}^{2}}{s}\right) \int d \Gamma_{2}\left|\mathcal{M}_{\mathrm{Born}}(x s)\right|^{2},
$$

includes all-order photon radiation in the soft regime at leading-logarithmic approximation and, simultaneously, correctly describes collinear radiation of up to three photons in the hard regime. It does not account for the helicity-flip part $f^{-}$(23) of the fixed-order structure function; this may either be added separately or just be dropped since it is subleading in the leading-logarithmic approximation.

In this description of the collinear region, there is no explicit cutoff $\Delta E_{\gamma}$ involved, and collinear virtual photons connected to at least one incoming particle are included. The latter part is effectively smeared over small photon energies, such that the +-distribution singularity of the finite-order result is replaced by a power-like behavior with a finite limit for $x \rightarrow 1$. Stated differently, the cancellation of infrared singularities between virtual and real corrections is built-in (for collinear photons), so that the main source of negative event weights is eliminated.

\subsection{Matching with NLO}

We combine the ISR-resummed LO result with the additional NLO contributions described in the previous section. To achieve this, we first subtract from the effective squared matrix element, for each incoming particle, the contribution of one soft photon that is contained in the ISR structure function (and has already been accounted for in the soft-photon factor),

$$
f_{\text {soft,ISR }}\left(\Delta E_{\gamma}, \Delta \theta_{\gamma}, m_{e}^{2}\right)=\frac{\eta}{4} \int_{x_{0}}^{1} d x\left(\frac{1+x^{2}}{1-x}\right)_{+}=\frac{\eta}{4}\left(2 \ln \left(1-x_{0}\right)+x_{0}+\frac{1}{2} x_{0}^{2}\right) .
$$

Here, $\eta$ is defined in Eq. (22), and the +-distribution is represented, e.g., by

$$
g(x)_{+}=\lim _{\epsilon \rightarrow 0}\left[\theta(1-x-\epsilon) g(x)-\delta(1-x-\epsilon) \int_{0}^{1-\epsilon} g(y) d y\right] .
$$

After this subtraction we have

$$
\begin{aligned}
\left|\widetilde{\mathcal{M}}_{\text {eff }}\left(\hat{s} ; \Delta E_{\gamma}, \Delta \theta_{\gamma}, m_{e}^{2}\right)\right|^{2}= & {\left[1+f_{\text {soft }}\left(\frac{\Delta E_{\gamma}}{\lambda}\right)-2 f_{\text {soft }, \text { ISR }}\left(\Delta E_{\gamma}, \Delta \theta_{\gamma}, \frac{m_{e}^{2}}{s}\right)\right]\left|\mathcal{M}_{\text {Born }}(\hat{s})\right|^{2} } \\
& +2 \operatorname{Re}\left[\mathcal{M}_{\text {Born }}(\hat{s}) \mathcal{M}_{\text {1-loop }}\left(\hat{s}, \lambda^{2}, m_{e}^{2}\right)\right]
\end{aligned}
$$

with $\hat{s}$ being the c.m. energy after radiation. This expression contains the Born term, the virtual and soft collinear contribution with the leading-logarithmic part of virtual photons and soft collinear emission removed, and soft non-collinear radiation of one photon; it still depends of the cutoff $\Delta E_{\gamma}$. Convoluting this with the resummed ISR structure function,

$$
\begin{aligned}
& \sigma_{\mathrm{v}+\mathrm{s}, \mathrm{ISR}}\left(s, \Delta E_{\gamma}, \Delta \theta_{\gamma}, m_{e}^{2}\right) \\
& =\int d x_{1} f_{\mathrm{ISR}}\left(x_{1} ; \Delta \theta_{\gamma}, \frac{m_{e}^{2}}{s}\right) \int d x_{2} f_{\mathrm{ISR}}\left(x_{2} ; \Delta \theta_{\gamma}, \frac{m_{e}^{2}}{s}\right) \int d \Gamma_{2}\left|\widetilde{\mathcal{M}}_{\mathrm{eff}}\left(\hat{s} ; \Delta E_{\gamma}, \Delta \theta_{\gamma}, m_{e}^{2}\right)\right|^{2},
\end{aligned}
$$



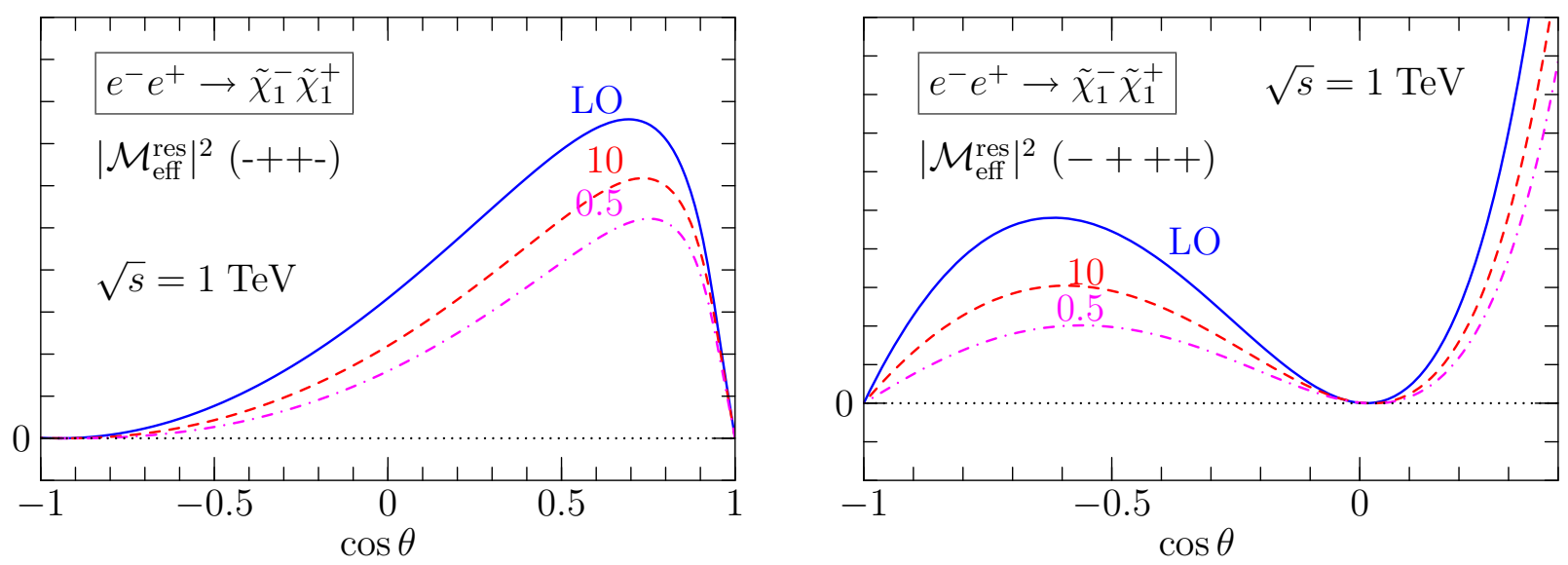

Figure 4: Effective squared matrix element (arbitrary units) with the one-photon ISR part subtracted, for $e^{-} e^{+} \rightarrow \tilde{\chi}_{1}^{-} \tilde{\chi}_{1}^{+}$as a function of the polar scattering angle $\theta$ at $\sqrt{s}=1 \mathrm{TeV}$. Left figure: Helicity combination -++- ; right figure: -+++ . Solid line: Born term; dashed: including virtual and soft contributions for $\Delta E_{\gamma}=10 \mathrm{GeV}$; dotted: same with $\Delta E_{\gamma}=0.5 \mathrm{GeV}$. The collinear cutoff is fixed at $\Delta \theta_{\gamma}=1^{\circ}$.

we obtain a modified $2 \rightarrow 2$ part of the total cross section. Note that this convolution also includes soft and collinear photonic corrections to the Born/one-loop interference. The complete result again contains in addition the $2 \rightarrow 3$ part,

$$
\sigma_{\text {tot,ISR }}\left(s, m_{e}^{2}\right)=\sigma_{\mathrm{v}+\mathrm{s}, \mathrm{ISR}}+\int_{\Delta E_{\gamma}, \Delta \theta_{\gamma}} d \Gamma_{3}\left|\mathcal{M}_{2 \rightarrow 3}(s)\right|^{2} .
$$

\subsection{Simulation}

As can be verified in Fig. 4, the resummation approach does eliminate the problem of negative weights: shifting the energy cutoff below the experimental resolution, e.g., $\Delta E_{\gamma}=0.5 \mathrm{GeV}$, such that photons are explicitly generated whenever they can be resolved, the subtracted effective squared $2 \rightarrow 2$ matrix element is still positive semidefinite in the whole phase space. Since neither the inclusion of the ISR structure function nor the addition of the $2 \rightarrow 3$ part introduces further sources of negative weights, unweighting of generated events is now possible, so this method allows for realistic simulation at NLO.

After resummation, the only potentially remaining source of negative event weights is the soft-noncollinear region. Negative weights are absent as long as

$$
\mathcal{O}(1) \times \frac{\alpha}{\pi} \ln \frac{\left(\Delta E_{\gamma}\right)^{2}}{s} \ln \left(\Delta \theta_{\gamma}\right)
$$

stays smaller than one, where the $\mathcal{O}(1)$ prefactor depends on the specific process. For the cutoff and parameter ranges we are considering here, this condition is fulfilled. 
A final possible improvement is to also convolute the $2 \rightarrow 3$ part with the ISR structure function,

$$
\begin{aligned}
& \sigma_{\text {tot,ISR }+}\left(s, m_{e}^{2}\right) \\
& =\int d x_{1} f_{\mathrm{ISR}}\left(x_{1} ; \Delta \theta_{\gamma}, \frac{m_{e}^{2}}{s}\right) \int d x_{2} f_{\mathrm{ISR}}\left(x_{2} ; \Delta \theta_{\gamma}, \frac{m_{e}^{2}}{s}\right) \\
& \quad \times\left(\int d \Gamma_{2}\left|\widetilde{\mathcal{M}}_{\mathrm{eff}}\left(\hat{s} ; \Delta E_{\gamma}, \Delta \theta_{\gamma}, m_{e}^{2}\right)\right|^{2}+\int_{\Delta E_{\gamma}, \Delta \theta_{\gamma}} d \Gamma_{3}\left|\mathcal{M}_{2 \rightarrow 3}(\hat{s})\right|^{2}\right) .
\end{aligned}
$$

This introduces another set of higher-order corrections, namely those where after an arbitrary number of collinear photons, one hard non-collinear photon is emitted. This additional resummation does not double-count. It catches logarithmic higher-order contributions where ordering in transverse momentum can be applied. Other, logarithmically subleading contributions are missed; this is consistent since the genuine second-order part is not calculated anyway.

\section{Results}

\subsection{Choosing Cutoffs}

In the kinematical ranges below the soft and collinear cutoffs, several approximations are made. In particular, the method neglegts contributions proportional to positive powers of $\Delta E_{\gamma}$ and $\Delta \theta_{\gamma}$, so the cutoffs must not be increased into the region where these effects could become important. On the other hand, decreasing cutoffs too much we can enter a region where the limited machine precision induces numerical instabilities. Therefore, we have to check the dependence of the total cross section as calculated by adding all pieces and identify parameter ranges for $\Delta E_{\gamma}$ and $\Delta \theta_{\gamma}$ where the result is stable but does not depend significantly on the cutoff values.

\section{Energy cutoff dependence}

In Fig. 5 we compare the numerical results obtained using the semianalytic fixed-order calculation with our Monte-Carlo integration in the fixed-order and in the resummation schemes, respectively. Throughout this section, we set the process energy to $\sqrt{s}=1 \mathrm{TeV}$ and refer to the SUSY parameter point SPS1a'. All $2 \rightarrow 2$ and $2 \rightarrow 3$ contributions are included, so the results would be cutoff-independent if there were no approximations involved.

The fixed-order Monte-Carlo result agrees with the semianalytic result, as it should be the case, as long as the cutoff is greater than a few GeV. For smaller cutoff values the Monte-Carlo result drastically departs from the semianalytic one because the virtual correction exceeds the LO term there, and therefore the $2 \rightarrow 2$ effective squared matrix element becomes negative in part of phase space. For the Monte-Carlo approach, aiming at unweighting events, the integrand is set to zero in regions where it is actually negative, and the result overshoots when this happens. 


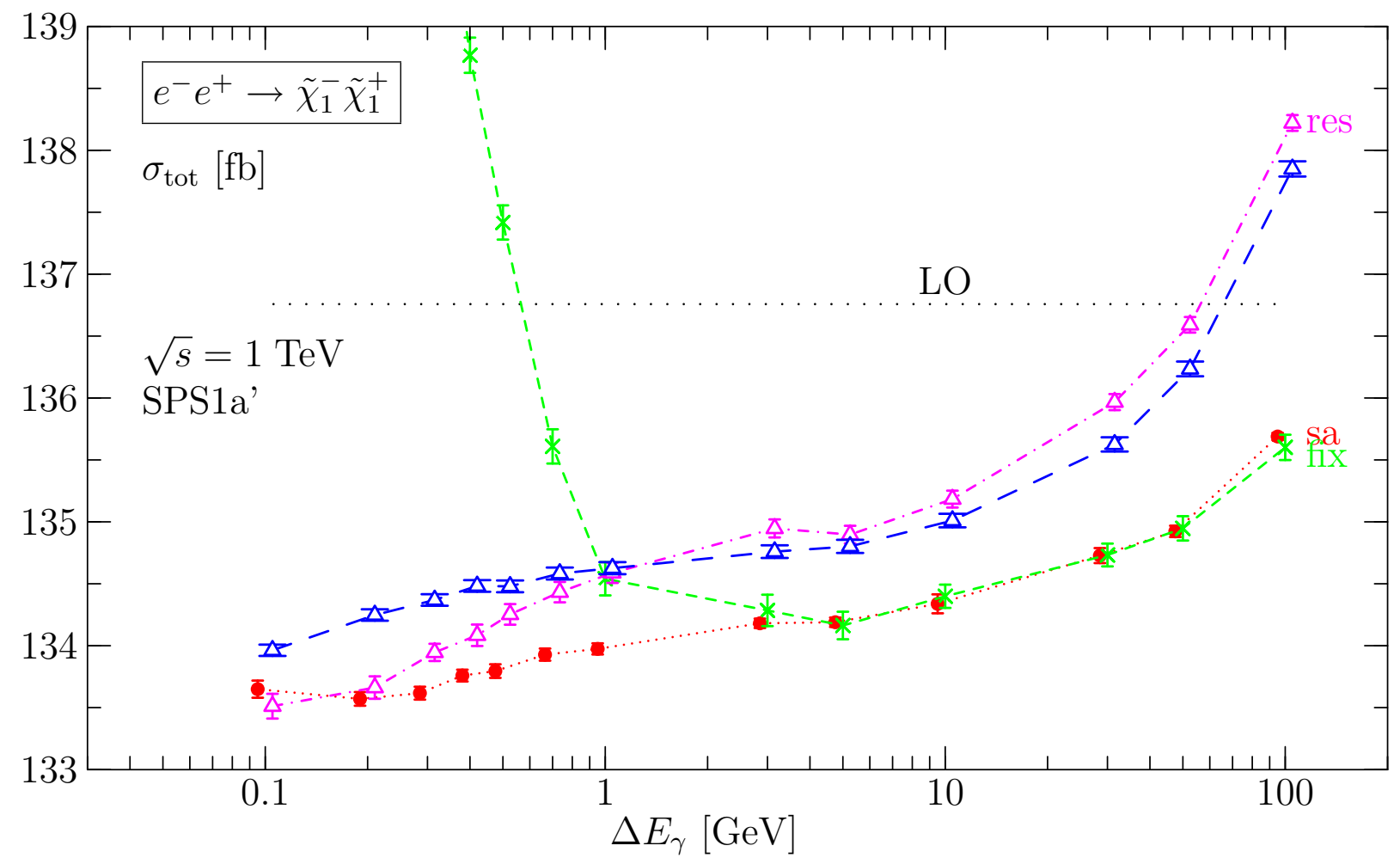

Figure 5: Total cross section dependence on the energy cutoff $\Delta E_{\gamma}$ using different calculational methods: 'sa' (dotted) = fixed-order semianalytic result [15] using FeynArts/FormCalc; 'fix' (dashed) = fixed-order Monte-Carlo result (8) using WHIZARD; 'res' (long-dashed) $=I S R$ resummed Monte-Carlo result (18) using WHIZARD; (dash-dotted) = same but resummation applied only to the $2 \rightarrow 2$ part (17). Statistical Monte-Carlo integration errors are shown. For the Monte-Carlo results, the collinear cutoff has been fixed to $\Delta \theta_{\gamma}=1^{\circ}$. The Born cross section is indicated by the dotted horizontal line.

The semianalytic fixed-order result is not exactly cutoff-independent, as one could naively expect. Instead, it exhibits a slight rise of the calculated cross section with increasing cutoff; for $\Delta E_{\gamma}=1 \mathrm{GeV}(10 \mathrm{GeV})$ the shift is about $2 \%$ ( $5 \%$ ) of the total cross section, respectively. While for cutoff values approaching the process energy the soft approximation is expected to fail, the rise at small cutoff values is due to the fact that in the soft-photon factor $f_{\text {soft }}\left(\frac{\Delta E_{\gamma}}{\lambda}\right)$ the kinematics is slightly tweaked (necessary to cancel the photon-mass dependence in the virtual part), and the cancellation of the logarithmic singularity in $\Delta E_{\gamma}$ is therefore not exact. The mismatch is parameterically of the order $\Delta E_{\gamma} / \sqrt{s}$ multiplied by the virtual correction; given the fact that the virtual correction exceeds the LO term at about $\Delta E_{\gamma}=1 \mathrm{GeV}$, we expect an error of up to a few $\%$ at that point. For $E \lesssim 0.1 \mathrm{GeV}$, this error becomes truly negligible.

The fully resummed result (18) shows an increase of about $5 \%$ of the total cross section with respect to the fixed-order result which stays roughly constant until $\Delta E_{\gamma}>10 \mathrm{GeV}$ where 


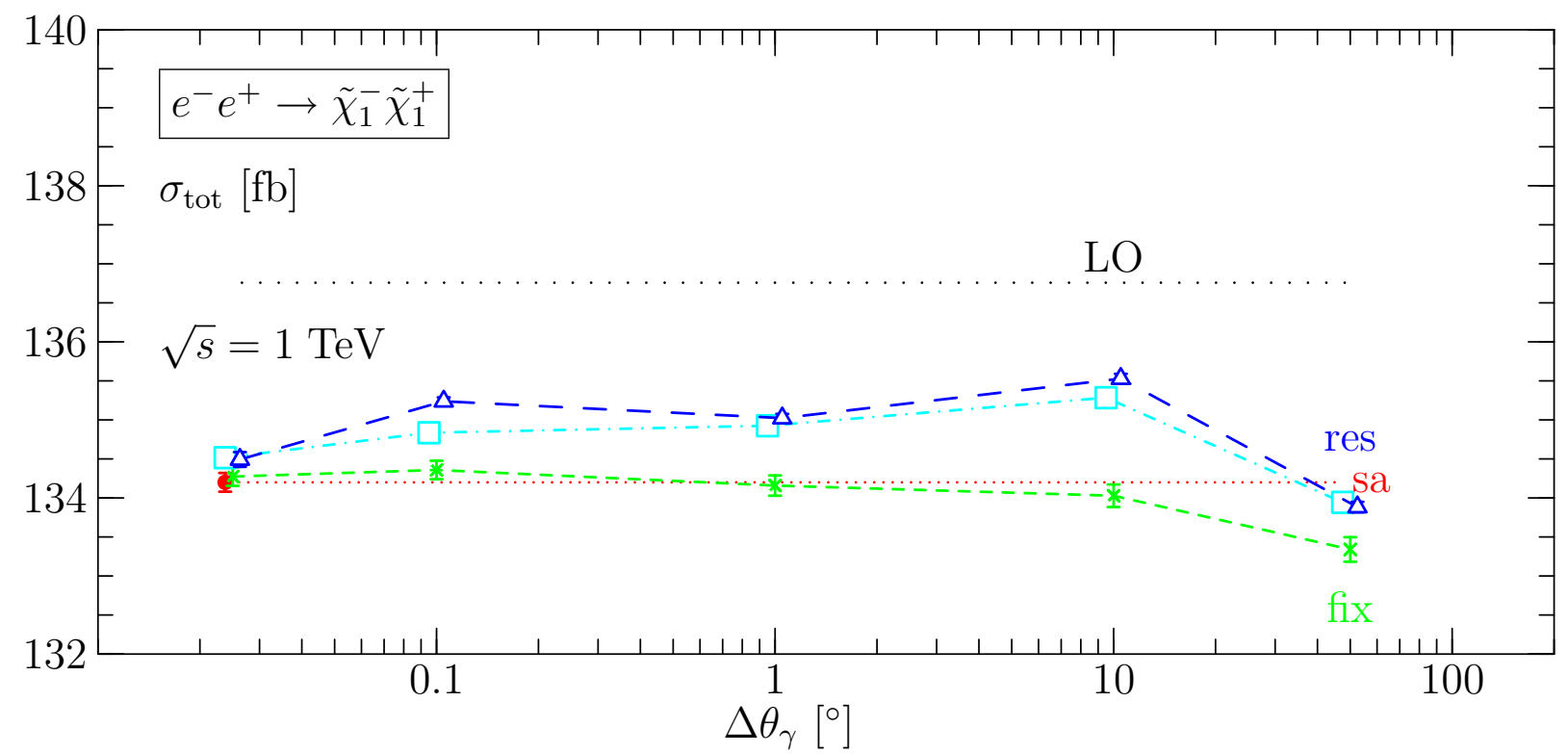

Figure 6: Total cross section dependence on the collinear cutoff $\Delta \theta_{\gamma}$ using three different calculational methods: sa (dotted) = fixed-order semianalytic result [15] using FeynArts/FormCalc; fix $($ dashed $)=$ fixed-order Monte-Carlo result (8) using WHIZARD; res (long-dashed) $=$ thirdorder ISR-resummed Monte-Carlo result (18) using WHIZARD; (dash-dotted) = same, but using the first-order ISR-resummed structure function instead. Statistical Monte-Carlo integration errors are shown. The soft cutoff has been fixed to $\Delta E_{\gamma}=10 \mathrm{GeV}$.

the soft approximation breaks down. This increase is a real effect; it is due to higher-order photon radiation that is absent from the fixed-order calculation. Comparing with the curve for $2 \rightarrow 2$ resummation (17), we observe that for $\Delta E_{\gamma}>1 \mathrm{GeV}$ these higher-order contributions are caught by ISR resummation of the $2 \rightarrow 2$ part, but are transferred to the $2 \rightarrow 3$ part if the cutoff is lowered further, i.e., one radiated photon is resolved.

At the ILC, with a cross section of more than $100 \mathrm{fb}$ and an integrated luminosity of $1 \mathrm{ab}^{-1}$, a statistical $1 \sigma$ fluctuation level of $2.5 \%$ is reached. Although systematical uncertainties in the analysis are likely to be relevant as well, the theoretical prediction of the Monte-Carlo generator should aim at matching that precision. To get rid of artefacts of the soft approximation at the level of $2 \%$, we have to choose $\Delta E_{\gamma} \leq 0.5 \mathrm{GeV}$. (For cutoffs lower than $0.1 \mathrm{GeV}$ doubleprecision numerics breaks down, although we still could obtain results by switching to quadruple precision.) Resumming photons leads to an increase of $5 \%$.

A common practice is to just convolute the Born part with the ISR structure function, leaving all other contributions at fixed $O(\alpha)$. Our results show that this is insufficient if the achievable accuracy is to be exploited.

To conclude, for the resummation method indicated by Eq. (18) the desired low cutoff values are actually acceptable, and the systematic errors induced by the soft and collinear 
approximations can be suppressed down to the expected level of statistical fluctuations. In principle, NNLO corrections and higher order effects of running couplings should be studied for a final verdict on the theoretical accuracy. However, we do not expect these corrections to be significant; in particular, at ILC energies electroweak Sudakov logarithms are still sufficiently small [27]. Off-shell and finite-width effects can be taken into account by interfacing the results obtained here with the multi-particle event generators presented in 12 .

\section{Collinear cutoff dependence}

The collinear cutoff $\Delta \theta_{\gamma}$ separates the region where, in the collinear approximation, higherorder radiation is resummed from the region where only a single photon is included, but treated exactly. We show the dependence of the result on this cutoff is in Fig. 6.

The plot shows that the main higher-order effect is associated with photon emission angles below $0.1^{\circ}$. Cutoff values between $0.1^{\circ}$ and $10^{\circ}$ are essentially equivalent. To achieve this cutoff-independence, collinear terms have to be included in the structure function beyond first order (up to third order in our case); using the first-order ISR-resummed structure function instead would miss some radiation at low angles $\theta_{\gamma}<1^{\circ}$, cf. the small difference at $\Delta \theta_{\gamma}=0.1^{\circ}$ between the first- and third-order results in Fig. 6] For $\theta_{\gamma}>10^{\circ}$, the collinear approximation breaks down.

\section{Photon mass dependence}

The dependence on the fictitious photon mass $\lambda$ is eliminated by implementing the soft-photon factor $f_{\text {soft }}$ before any further manipulations are done. Therefore, while the photon mass remains a parameter in the matrix element code, the result does not numerically depend on it, regardless which method has been chosen.

\subsection{Energy Dependence of the Total Cross Section}

Fixing the cutoffs to reasonable values, we can use the integration part of the Monte-Carlo generator to evaluate the total cross section at NLO for various energies. This is shown in Fig. [7, where we display the LO result together with the NLO result for the fixed-order and resummed approach indicated above, respectively. Near the cross-section maximum, the relative correction in the fixed-order (resummed) approach is about $-5 \%$, approaching $-2 \%(-1.5 \%)$ at $\sqrt{s}=1 \mathrm{TeV}$, respectively. Near threshold and at asymptotic energies, the relative NLO correction is larger in magnitude.

\subsection{Simulated Distributions}

The strength of the Monte-Carlo method lies not in the ability to calculate total cross sections, but to simulate physical event samples. We have used the WHIZARD event generator augmented by the effective matrix elements and structure functions as introduced above, to generate unweighted event samples for chargino production. 

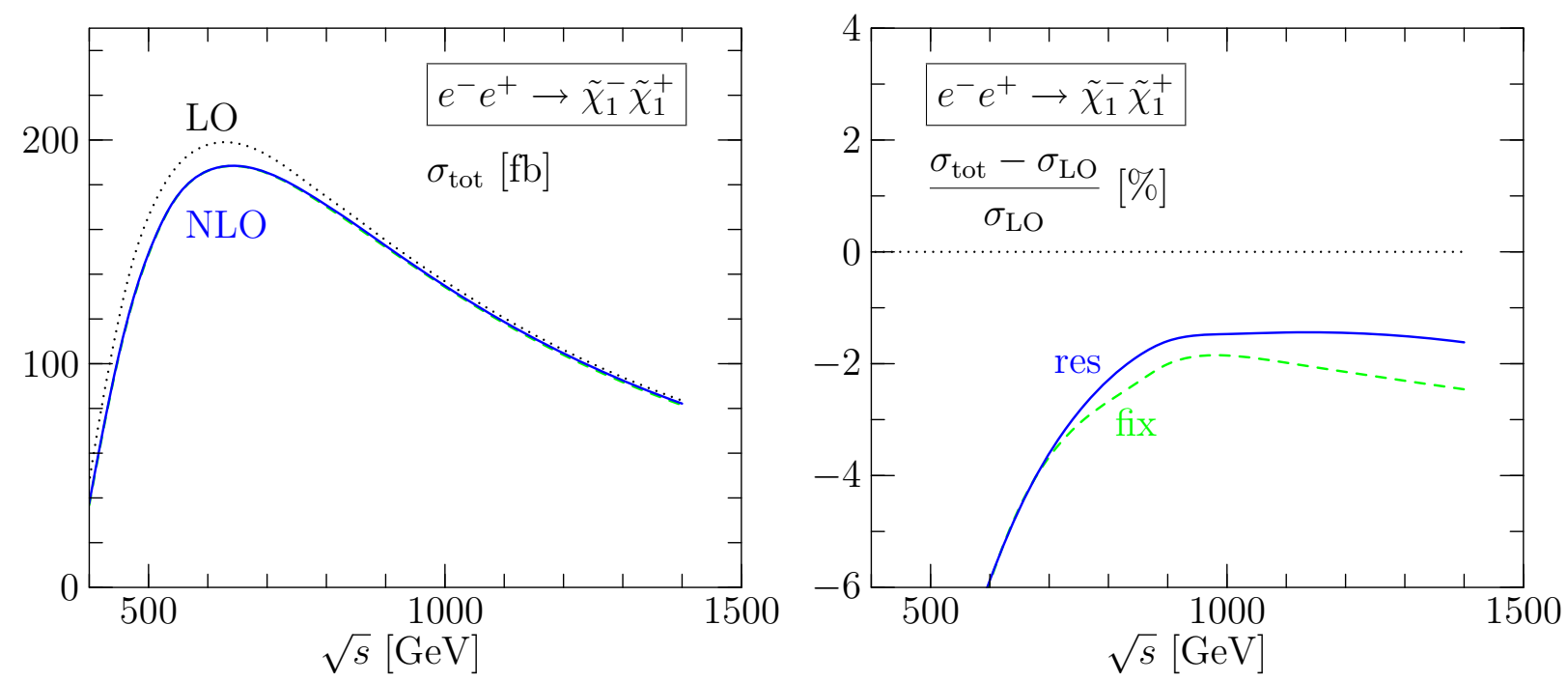

Figure 7: Left: $\sigma_{\text {tot }}$ in $f b$ as a function of $\sqrt{s}$; right: relative correction to the Born term in percent. $L O($ dotted) $=$ Born cross section without ISR; NLO/fix (dashed) $=$ fixed-order approach; $N L O /$ res (full) $=$ resummation approach. We choose $\Delta E_{\gamma}=0.005 \sqrt{s}$ and $\Delta \theta_{\gamma}=1^{\circ}$ as cutoffs separating the hard (non-collinear) from the soft (collinear) regions, respectively.
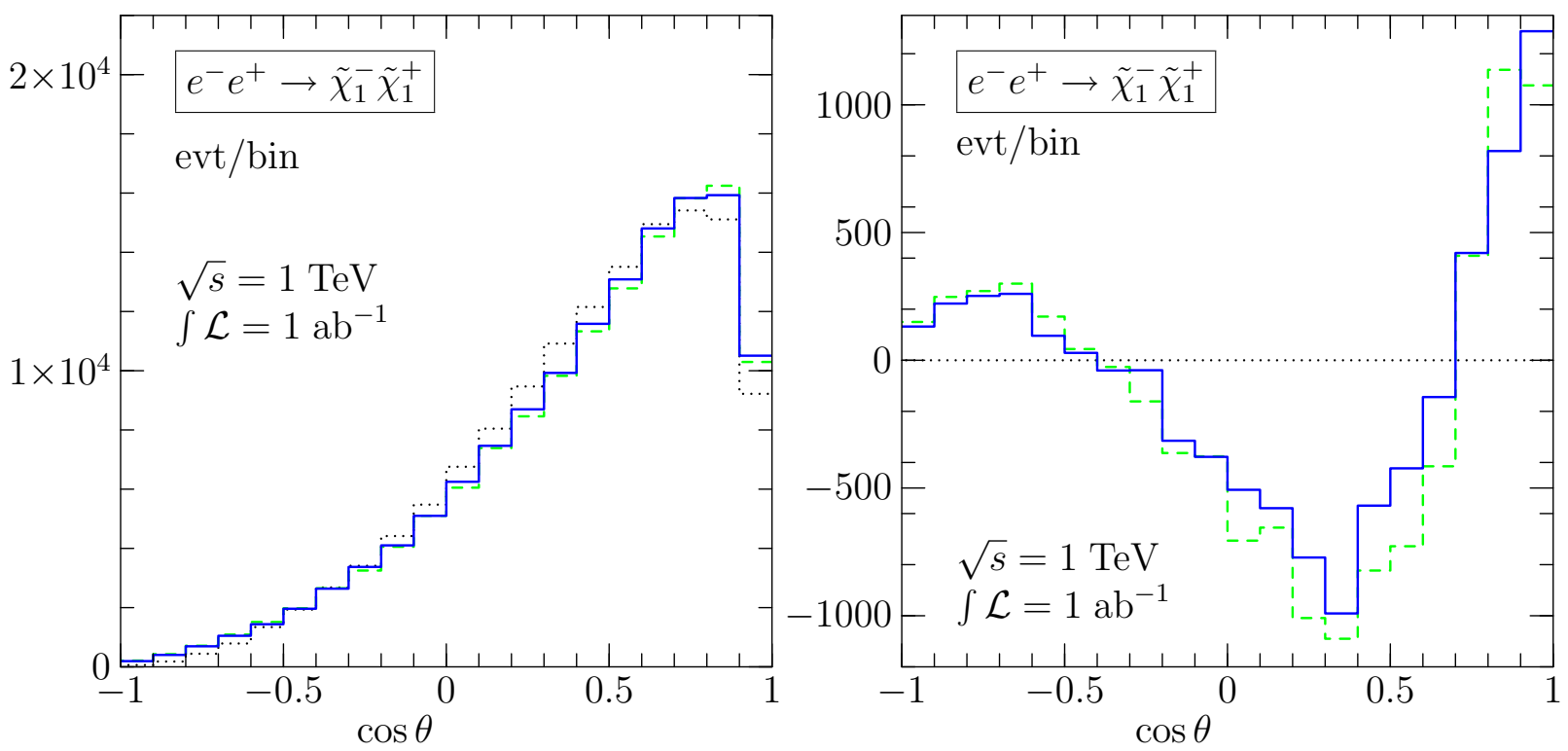

Figure 8: Polar scattering angle distribution for an integrated luminosity of $1 \mathrm{ab}^{-1}$ at $\sqrt{s}=$ $1 \mathrm{TeV}$. Left: total number of events per bin; right: difference w.r.t. the Born distribution. LO $($ dotted $)=$ Born cross section without ISR; fix (dashed) $=$ fixed-order approach; res (full) $=$ resummation approach. Cutoffs: $\Delta E_{\gamma}=3 \mathrm{GeV}$ and $\Delta \theta_{\gamma}=1^{\circ}$. 
To evaluate the importance of the NLO improvement, in Fig. 8 we show the binned distribution of the chargino production angle as obtained from a sample of unweighted events corresponding to $1 \mathrm{ab}^{-1}$ of integrated luminosity. The collider energy has been set to $1 \mathrm{TeV}$, the SUSY parameter point SPS1a' is the same one as for the previous plots. With cutoffs $\Delta \theta_{\gamma}=1^{\circ}$ and $\Delta E_{\gamma}=3 \mathrm{GeV}$ we are not far from the expected experimental resolution, while for the fixed-order approach negative event weights do not yet pose a problem. (As discussed above, for the resummation approach decreasing cutoffs further is possible and preferred, but choosing lower values would invalidate the fixed-order approach for the comparison.)

The histograms illustrate the fact that NLO corrections in chargino production are not just detectable, but rather important for an accurate prediction, given the high ILC luminosity. The correction cannot be approximated by a constant $K$-factor but takes a different shape than the LO distribution. The correction is positive in the forward and backward directions, but negative in the central region. The effects of photon resummation are not as striking, but still statistically significant; they are visible mostly in the central-to-forward region. Apparently, to carefully choose the resummation method and cutoffs will be critical for a truly precise analysis of real ILC data.

\section{$5 \quad$ Summary and Outlook}

We have presented results obtained from implementing NLO corrections into a Monte-Carlo event generator for chargino pair-production at the ILC. On top of the genuine SUSY/electroweak corrections, we have considered several approaches of including photon radiation, where a strict fixed-order approach allows for comparison and consistency checks with published semianalytic results in the literature, while a version with soft- and hard-collinear resummation of photon radiation not just improves the numerical result, but actually is more straightforward to implement and does not suffer from negative event weights in or near the accessible part of phase space.

A careful analysis of the dependence on the technical cutoffs on photon energy and angle that slice phase space in regions, reveals uncertainties related to higher-order radiation and breakdown of the soft or collinear approximations. For the level of precision required by ILC analyses, the cutoffs have to be chosen rather low. Resummation of photon radiation is required not just for precision, but also to get rid of negative event weights in the simulation. In the Monte-Carlo event generator WHIZARD we have thus implemented the NLO result with higherorder resummation in critical regions. The generator accounts for all yet known higher-order effects, allows for cutoffs small enough that soft- and collinear-approximation artefacts are negligible, and explicitly generates photons where they can be resolved experimentally.

The generator that we have constructed should be regarded as a step towards complete NLO simulation of SUSY processes at the ILC. If charginos happen to be metastable, it already provides all necessary ingredients. Beam effects (beamstrahlung and energy spread, polarization) are available for simulation and can easily be included. However, charginos are metastable only for peculiar SUSY parameter points; in general we have to take into account chargino decay and the corresponding additional NLO corrections. These we have to match with off-shell and 
background effects, already available for simulation in WHIZARD. Furthermore, in the threshold region the Coulomb singularity calls for resummation, not yet accounted for in the program. These lines of improvement will be pursued in future work.

\section{Acknowledgments}

We are grateful to T. Hahn, W. Hollik and T. Fritzsche for valuable discussions, technical help, and for providing us code prior to publication. We furthermore acknowledge S. Dittmaier for helpful discussions, and T. Ohl for a careful reading of the manuscript. This work was supported by the German Helmholtz Association, Grant VH-NG-005. 


\section{A Soft and collinear photon factors}

The soft-photon factor that allows us to eliminate the ficticious photon mass $\lambda$ in favor of a physical photon-energy cutoff $\Delta E_{\gamma}$ is given by the integral [28,22, 29]

$$
f_{\text {soft }}=-\frac{\alpha}{2 \pi} \sum_{i, j=e^{ \pm}, \tilde{\chi}^{ \pm}} \int_{|\mathbf{k}| \leq \Delta \mathbf{E}_{\gamma}} \frac{d^{3} k}{2 \omega_{k}} \frac{( \pm) p_{i} p_{j} Q_{i} Q_{j}}{\left(p_{i} k\right)\left(p_{j} k\right)} .
$$

$p_{i}(k)$ denote the electron/chargino (photon) four-vectors, respectively, while

$$
\omega_{k}=\sqrt{\mathbf{k}^{2}+\lambda^{2}}
$$

is the energy of a photon regularized by the photon mass $\lambda . Q_{i}$ are the corresponding charges. The sign is - for incoming and + for outgoing particles, respectively.

For conserved helicity, the collinear radiation of one photon can be approximated by convoluting the no-photon matrix element with the structure function (see, e.g., [30])

$$
f^{+}(x)=\frac{\eta}{4} \frac{1+x^{2}}{(1-x)} .
$$

Here, with $k_{\max }^{\top}=p_{e}^{0} \Delta \theta_{\gamma}$ being the collinear cutoff, the expansion parameter $\eta$ is defined as

$$
\eta=\frac{2 \alpha}{\pi}\left[\ln \left(\frac{s}{4 m_{e}^{2}}\left(\Delta \theta_{\gamma}\right)^{2}\right)-1\right]
$$

The helicity-flip structure function,

$$
f^{-}(x)=\frac{\alpha}{2 \pi}(1-x)
$$

does not contain a logarithmic enhancement with $\Delta \theta_{\gamma}$, so the helicity-flip part of collinear radiation is subdominant.

In the soft-collinear region $x \approx 1, \theta_{\gamma} \approx 0$, the leading logarithms in $\Delta \theta_{\gamma}$ (i.e., powers of $\eta$ ) can be resummed to all orders [25]. Matching this with the complete $x$-dependent expressions for hard collinear radiation to first, second, and third order in $\eta$, Skrzypek and Jadach obtained the ISR structure function [26]

$$
\begin{aligned}
f_{\mathrm{ISR}}(x)= & \frac{\exp \left(-\frac{1}{2} \eta \gamma_{E}+\frac{3}{8} \eta\right)}{\Gamma\left(1+\frac{\eta}{2}\right)} \frac{\eta}{2}(1-x)^{\left(\frac{\eta}{2}-1\right)}-\frac{\eta}{4}(1+x) \\
+ & \frac{\eta^{2}}{16}\left(-2(1+x) \ln (1-x)-\frac{2 \ln x}{1-x}+\frac{3}{2}(1+x) \ln x-\frac{x}{2}-\frac{5}{2}\right) \\
+ & \frac{\eta^{3}}{8}\left[-\frac{1+x}{2}\left(\frac{9}{32}-\frac{\pi^{2}}{12}+\frac{3}{4} \ln (1-x)+\frac{1}{2} \ln ^{2}(1-x)-\frac{1}{4} \ln x \ln (1-x)\right.\right. \\
& \left.+\frac{1}{16} \ln ^{2} x-\frac{1}{4} \operatorname{Li}_{2}(1-x)\right) \\
& +\frac{1+x^{2}}{2(1-x)}\left(-\frac{3}{8} \ln x+\frac{1}{12} \ln ^{2} x-\frac{1}{2} \ln x \ln (1-x)\right) \\
& \left.-\frac{1}{4}(1-x)\left(\ln (1-x)+\frac{1}{4}\right)+\frac{1}{32}(5-3 x) \ln x\right] .
\end{aligned}
$$




\section{B Two-Photon Phase-Space Regimes in the Resumma- tion Method}

The class of processes we are considering exhibits the usual infrared and collinear (for $m_{e}=0$ ) singularities in individual contributions to the physical result, which we treat by standard methods. In the fixed-order approach where only one photon is present, the consequences of this phase-space slicing are evident: (i) In the soft region, the photon energy is set to zero in the matrix element, and the real contribution is cancelled against the IR-divergent part of the virtual correction, neglecting corrections proportional to the photon energy. (ii) In the hard-collinear region, the photon-emission part of the matrix element is replaced by a structure function, neglecting corrections proportional to the separation angle. As long as the problem of negative event weights can be ignored, and numerics is not an issue, the description at fixed NLO always improves if any of those cutoffs are lowered.

However, the resummation method described in Sec. 3 involves all orders of soft-photon radiation. Shifting cutoffs changes the type of higher-order contributions that are included, so lowering cutoffs not necessarily improves the description.

To clarify this issue, let us focus on the $O\left(\alpha^{2}\right)$ correction, i.e., two photons (real or virtual). Since we do not consider two-loop diagrams, this correction is not completely accounted for, but dominant contributions are included.

In the resummation method, there are three different ways of dealing with real and virtual photons:

(a) soft approximation [29]:

describes collinear and non-collinear soft photons; neglects contributions $\propto \frac{\Delta E_{\gamma}}{\sqrt{s}}$; is combined in the sequel with the soft photonic part of the one-loop matrix element

(b) ISR [26]:

describes collinear real and virtual photons; neglects interference terms in photon emissions. Assumes $k^{\top}$-ordering of the emitted photons, i.e. for $j>i$ : $k_{j}^{\top}>k_{i}^{\top}$, and in $n^{t h}$ order: $\sum_{i=1}^{n} k_{i}^{\top}<k_{\max }^{\top}$, where $k_{\max }^{\top}$ is fixed.

(c) real emission given by exact (hard non-collinear) matrix element $\mathcal{M}_{2 \rightarrow 3}$

Considering now the treatment of two photons (i.e. $\mathcal{O}\left(\alpha^{2}\right)$ corrections), at least one of the photons is always described by the ISR structure function. But when the Born term is convoluted with the ISR function, there are also two-photon contributions described solely by the ISR. We have to distinguish between the cases where (i) the two photons are attached to the same or (ii) to different incoming particles. In case (i), we consider the three terms

$$
\mathcal{O}\left(\alpha^{2}\right)_{\mathrm{ISR}}-\mathcal{O}(\alpha)_{\mathrm{ISR}} \mathcal{O}(\alpha)_{\mathrm{ISR}}^{\mathrm{soft}}+\mathcal{O}(\alpha)_{\mathrm{ISR}} \mathcal{O}(\alpha)_{\text {soft }}
$$

The first term contains all pairs of collinear photons from the ISR, $k^{\top}$-ordered; the last term contains a first photon from ISR and a second one from the soft-photon factor (SPF, which in the following is understood to include the soft photonic one-loop contribution). The term in the 
middle is the subtraction to avoid double-counting of soft photons described in subsection 3.2 . Here both photons are from the ISR, the first one with arbitrary energy, the second one soft.

If the second of the considered photons is soft, and both are $k^{\top}$-ordered, then there is an exact cancellation between the first two terms. For non $k^{\top}$-ordered photons, the first term gives no contribution, and there is a cancellation between the second and third term, which results in a difference between the "exact" SPF expression and the ISR LLA term for the incoming particle \# $j$ :

$$
\Delta_{j}=\mathcal{O}(\alpha)_{j, \text { soft }}-\mathcal{O}(\alpha)_{j, \text { ISR }}
$$

In the case (ii), we write the terms schematically as

$$
\begin{aligned}
& \mathcal{O}(\alpha)_{1, \mathrm{ISR}} \mathcal{O}(\alpha)_{2, \mathrm{ISR}} \\
& +\mathcal{O}(\alpha)_{1, \mathrm{ISR}}\left(\mathcal{O}(\alpha)_{2, \mathrm{soft}}-\mathcal{O}(\alpha)_{2, \mathrm{ISR}}^{\text {soft }}\right) \\
& +\left(\mathcal{O}(\alpha)_{1, \mathrm{soft}}-\mathcal{O}(\alpha)_{1, \mathrm{ISR}}^{\text {soft }}\right) \mathcal{O}(\alpha)_{2, \mathrm{ISR}}
\end{aligned}
$$

Since here there are always two different structure functions involved, $k^{\top}$-ordering is absent, and after a cancellation of soft terms one is left with

$$
\Delta_{1} \mathcal{O}(\alpha)_{2, \mathrm{ISR}}+\mathcal{O}(\alpha)_{1, \mathrm{ISR}} \Delta_{2}+\mathcal{O}(\alpha)_{1, \mathrm{ISR}} \mathcal{O}(\alpha)_{2, \mathrm{ISR}}
$$

which is up to the missing terms $\Delta_{1} \Delta_{2}$ equivalent to an SPF description for both legs.

We now investigate the changes induced by raising one of the two cutoffs. Generally, for the contributions with two real photons, we loose contributions if we lower the cutoffs since double photon radiation is not accounted for by the $2 \rightarrow 3$ matrix element. Convoluting also the $2 \rightarrow 3$ matrix element with the ISR structure function as proposed in Equ. (18) gives contributions with a collinear and hard non-collinear photon and cures the problem. But still, raising the cutoff $\Delta \theta_{\gamma}$ opens up phase space for the first photon. Thus, raising the cutoffs gives a better description of these contributions as long as the collinear approximation is valid. The same holds for the energy cutoff, but we see from Figs. 5 and 6 that the soft approximation fails much earlier than the collinear description.

By including the next order of real photon radiation explicitly, i.e.,

$$
\int_{\Delta E_{\gamma, i}, \Delta \theta_{\gamma, i}} d \Gamma_{4}\left|\mathcal{M}_{2 \rightarrow 4}(s)\right|^{2},
$$

we can further improve the description of this part of two-photon phase space. This contribution, which is however tiny for the cutoff values considered here, can easily be added using WHIZARD as a tree-level event generator.

For completeness, we finally discuss the reshuffling of contributions in the overlap region of the soft-collinear and hard-collinear (ISR) descriptions. If we raise $\Delta E_{\gamma}$ while keeping $\Delta \theta_{\gamma}$ fixed, photons that have been hard now become soft. In the case (ii) (photons radiated from two different external particles), a photon which has been described by the structure function, comes now with the SPF. For the case (i), we have to distinguish whether the two photons are $k^{\top}$-ordered or not. If they are, the description again changes from the ISR to the SPF. If 
there is no $k^{\top}$-ordering, then the photons either change from hard+soft to soft+soft, which is a smooth transition where only the last two terms of Equ. (25) are involved, or the second photon changes to soft for the combinations hard+hard or soft+hard. In that case there appear new contributions of the form $\Delta \mathcal{O}(\alpha)_{\text {ISR }}$, which have not been there before.

Raising $\Delta \theta_{\gamma}$, while keeping $\Delta E_{\gamma}$ fixed, shuffles photons from a non-collinear to a collinear description. The interesting region is for photons that lie in the soft regime near the limit of the soft-collinear regime and change into the latter after raising the angle cutoff. For $k^{\top}$-ordered photons the cross-over is smooth and the second photon stays with the SPF, while for nonordered photons the description switches from the SPF to the the difference between ISR and SPF. For the case (ii) of radiation from different legs, the description always remains with the SPF.

For more details see 31 .

\section{SPS1a'}

The SUSY parameter point SPS1a' is defined in Ref. 3]; it is a SUGRA-type scenario derived from the parameter set

$$
\begin{gathered}
m_{0}=70 \mathrm{GeV}, \quad m_{1 / 2}=250 \mathrm{GeV}, \quad \tan \beta=10 \\
\mu>0, \quad A_{0}=-300 \mathrm{GeV}
\end{gathered}
$$

The precise spectrum and coupling parameters are computed using the renormalization-group evolution code of Ref. 32; the values can be found in Ref. [3]. The chargino masses and widths are also listed in Table 1 . 


\section{References}

[1] For reviews, see, e.g., H. P. Nilles, Phys. Rept. 110 (1984) 1; H. E. Haber and G. L. Kane, Phys. Rept. 117 (1985) 75; M. Drees, R. Godbole and P. Roy, "Theory and phenomenology of sparticles", World Scientific (2004).

[2] B. C. Allanach et al., in Proc. of the APS/DPF/DPB Summer Study on the Future of Particle Physics (Snowmass 2001) ed. N. Graf, Eur. Phys. J. C 25, 113 (2002) [eConf C010630, P125 (2001)] [arXiv:hep-ph/0202233.

[3] J. A. Aguilar-Saavedra et al., Eur. Phys. J. C 46, 43 (2006) [arXiv hep-ph/0511344].

[4] ATLAS TDR, report CERN/LHCC/99-15 (1999); CMS TP, report CERN/LHCC/94-38 (1994) and references therein.

[5] J.A. Aguilar-Saavedra et al. (ECFA/DESY LC Physics Working Group), TESLA: Technical design report. Part III: Physics at an $e^{+} e^{-}$Linear Collider, DESY-2001-011, hepph/0106315.

[6] G. Weiglein et al. [LHC/LC Study Group Collaboration], arXiv:hep-ph/0410364.

[7] B. C. Allanach et al., arXiv:hep-ph/0602198.

[8] S. Y. Choi, A. Djouadi, H. S. Song and P. M. Zerwas, Eur. Phys. J. C 8, 669 (1999) [arXiv:hep-ph/9812236. S. Y. Choi, A. Djouadi, M. Guchait, J. Kalinowski, H. S. Song and P. M. Zerwas, Eur. Phys. J. C 14, 535 (2000) [arXiv hep-ph/0002033.

[9] T. Sjostrand, S. Mrenna and P. Skands, JHEP 0605, 026 (2006) [arXiv hep-ph/0603175. G. Corcella et al., arXiv:hep-ph/0210213, F. E. Paige, S. D. Protopopescu, H. Baer and X. Tata, arXiv hep-ph/0312045; N. Ghodbane, arXiv hep-ph/9909499; E. Boos et al. [CompHEP Collaboration], Nucl. Instrum. Meth. A 534, 250 (2004) [arXiv hep-ph/0403113; J. Fujimoto, T. Ishikawa, M. Jimbo, T. Kaneko, T. Kon and M. Kuroda, arXiv:hep-ph/0402144.

[10] A. Denner, S. Dittmaier, M. Roth and D. Wackeroth, Nucl. Phys. B 587, 67 (2000) [arXiv hep-ph/0006307].

[11] A. Denner, S. Dittmaier, M. Roth and L. H. Wieders, Phys. Lett. B 612, 223 (2005) [arXiv hep-ph/0502063; A. Bredenstein, A. Denner, S. Dittmaier and M. M. Weber, arXiv:hep-ph/0604011.

[12] K. Hagiwara, W. Kilian, F. Krauss, T. Ohl, T. Plehn, D. Rainwater, J. Reuter, and S. Schumann, Phys. Rev. D 73, 055005 (2006) [arXiv hep-ph/0512260; J. Reuter et al., arXiv:hep-ph/0512012. 
[13] http://www-ttp.physik. uni-karlsruhe.de/whizard/; W. Kilian, WHIZARD 1.0: A generic Monte-Carlo integration and event generation package for multi-particle processes. Manual, LC-TOOL-2001-039; W. Kilian, WHIZARD: Complete simulations for electroweak multi-particle processes, in Proceedings of ICHEP, Amsterdam 2002, p. 831.

[14] O. Nicrosini and L. Trentadue, Phys. Lett. B 196, 551 (1987); G. Bonvicini and L. Trentadue, Nucl. Phys. B 323, 253 (1989).

[15] T. Fritzsche and W. Hollik, Nucl. Phys. Proc. Suppl. 135, 102 (2004) [arXiv hep-ph/0407095].

[16] T. Hahn, Comput. Phys. Commun. 140, 418 (2001) [arXiv hep-ph/0012260]. T. Hahn and C. Schappacher, Comput. Phys. Commun. 143, 54 (2002) [arXiv:hep-ph/0105349. T. Hahn and M. Perez-Victoria, Comput. Phys. Commun. 118, 153 (1999) [arXiv hep-ph/9807565].

[17] W. Öller, H. Eberl and W. Majerotto, Phys. Rev. D 71, 115002 (2005) [arXiv hep-ph/0504109.

[18] T. Ohl, O'Mega: An Optimizing Matrix Element Generator, in Proceedings of 7th International Workshop on Advanced Computing and Analysis Techniques in Physics Research (ACAT 2000) (Fermilab, Batavia, Il, 2000) [arXiv/hep-ph/0011243; M. Moretti, T. Ohl, J. Reuter, LC-TOOL-2001-040 [arXiv:hep-ph/0102195]; J. Reuter, hep-th/0212154.

[19] K. Hagiwara and D. Zeppenfeld, Nucl. Phys. B 274, 1 (1986).

[20] W. Kilian, T. Ohl, J. Reuter, O'Mega/Whizard: Multi-Purpose Multi-Particle Event Generator for the Standard Model, SUSY and Beyond, in preparation

[21] S. Dittmaier, Phys. Rev. D 59, 016007 (1999) [arXiv:hep-ph/9805445].

[22] M. Böhm, A. Denner and W. Hollik, Nucl. Phys. B 304, 687 (1988); F. A. Berends, R. Kleiss and W. Hollik, Nucl. Phys. B 304, 712 (1988).

[23] J. P. Alexander, G. Bonvicini, P. S. Drell and R. Frey, Phys. Rev. D 37, 56 (1988).

[24] S. Catani and M. H. Seymour, Phys. Lett. B 378, 287 (1996) [arXiv hep-ph/9602277; S. Catani, S. Dittmaier, M. H. Seymour and Z. Trocsanyi, Nucl. Phys. B 627, 189 (2002) [arXiv hep-ph/0201036].

[25] V. N. Gribov and L. N. Lipatov, Sov. J. Nucl. Phys. 15, 438 (1972) [Yad. Fiz. 15, 781 (1972)]; Sov. J. Nucl. Phys. 15, 675 (1972) [Yad. Fiz. 15, 1218 (1972)]; E. A. Kuraev and V. S. Fadin, Sov. J. Nucl. Phys. 41, 466 (1985) [Yad. Fiz. 41, 733 (1985)].

[26] M. Skrzypek and S. Jadach, Z. Phys. C 49, 577 (1991). 
[27] M. Beccaria, M. Melles, F. M. Renard, S. Trimarchi and C. Verzegnassi, Int. J. Mod. Phys. A 18, 5069 (2003).

[28] G. 't Hooft and M. J. G. Veltman, Nucl. Phys. B 153, 365 (1979).

[29] A. Denner, Fortsch. Phys. 41, 307 (1993).

[30] M. Böhm and S. Dittmaier, Nucl. Phys. B 409, 3 (1993); S. Dittmaier, RX-1526 (WURZBURG)

[31] T. Robens, PhD thesis, University of Hamburg, 2006 [in preparation].

[32] W. Porod, Comput. Phys. Commun. 153, 275 (2003) [arXiv:hep-ph/0301101. 